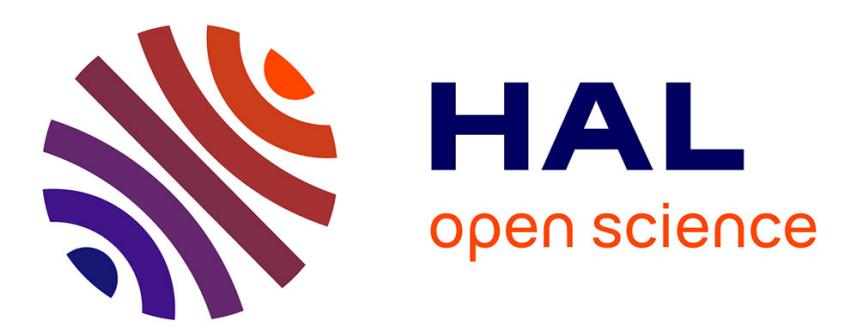

\title{
Numerical stability analysis and flow simulation of lid-driven cavity subjected to high magnetic field
}

Luca Marioni, François Bay, Elie Hachem

\section{To cite this version:}

Luca Marioni, François Bay, Elie Hachem. Numerical stability analysis and flow simulation of lid-driven cavity subjected to high magnetic field. Physics of Fluids, 2016, 28 (5), pp.057102. 10.1063/1.4948433 . hal-01311179

\section{HAL Id: hal-01311179 \\ https://hal.science/hal-01311179}

Submitted on 10 May 2016

HAL is a multi-disciplinary open access archive for the deposit and dissemination of scientific research documents, whether they are published or not. The documents may come from teaching and research institutions in France or abroad, or from public or private research centers.
L'archive ouverte pluridisciplinaire HAL, est destinée au dépôt et à la diffusion de documents scientifiques de niveau recherche, publiés ou non, émanant des établissements d'enseignement et de recherche français ou étrangers, des laboratoires publics ou privés. 


\title{
Numerical stability analysis and flow simulation of lid-driven cavity subjected to high magnetic field
}

\author{
L. Marioni ${ }^{1, *, \dagger}$, F. Bay ${ }^{1}$ and E. Hachem ${ }^{1}$ \\ ${ }^{1}$ MINES ParisTech, Center for Materials Forming (CEMEF), UMR CNRS 7635, BP 207, 06904 Sophia-Antipolis, \\ France.
}

\section{SUMMARY}

In this work we study the flow of a conducting fluid inside a two-dimensional square domain. The problem is solved by using a variational multiscale finite element approach. The study focuses on a high magnetic interaction parameter range and high Reynolds number. Under the imposition of a high magnetic field, the flow gets regularized, but fast transient phenomena take place, which could lead to numerical errors. An expression to compute the maximum time-step which guarantees convergence in explicit schemes is proposed and validated through numerical tests.

Received 14 December 2015; accepted 18 April 2016; published online May 2016

KEY WORDS: Magnetohydrodynamics, lid-driven cavity, braking, variational multiscale approach

This is the author's copy of the accepted manuscript. Please refer to:

$\ll$ L. Marioni, F. Bay, E. Hachem. Numerical Stability Analysis and Flow Simulation of Lid-Driven Cavity Subjected to High Magnetic Field. Physics of Fluids, 28, 057102, 2016.

doi: $10.1063 / 1.4948433 \gg$

for the edited/published version.

\section{INTRODUCTION}

The flow in a cavity induced by the motion of one of the walls is a classical benchmark for fluid mechanics analyses and it has been widely studied in literature $[1,2,3]$. The most interesting part of the flow is the area close to the corners, where high turbulence and discontinuous velocity occur [4]. The flow has been studied at different range of Reynolds number $(R e)$ up to 100000 in [5]. Approaches similar to the one used in the present work have been used to study Reynolds from 10000 in [6, 7] and up to 50000 in $[8,9]$. The number of induced eddies increases with the increasing of the Reynolds number, their size decreases and the flow turns to be chaotic [10]. This flow can be braked by the superimposition of a constant magnetic field [11]. This case has been used as benchmark for different applications, from biomagnetic fluids [12], to nanofluids [13]. The stabilisation due to the external magnetic field in the cavity has also been studied $[14,15,16]$. Deeper investigations have been done on the natural convected cavity under an external magnetic field both numerically $[17,18,19,20]$ and experimentally [21]. At the best of our knowledge, the flow behaviour at high Stuart number has not been object of studies. The idea is to use this benchmark to test the numerical coupling between the fluid flow and the magnetic field at different configurations. The framework of this work is braking application in material forming, in order to

\footnotetext{
${ }^{\dagger}$ E-mail: luca.marioni@mines-paristech.fr

${ }^{*}$ Correspondence to: Luca Marioni, Mines ParisTech, France.
} 

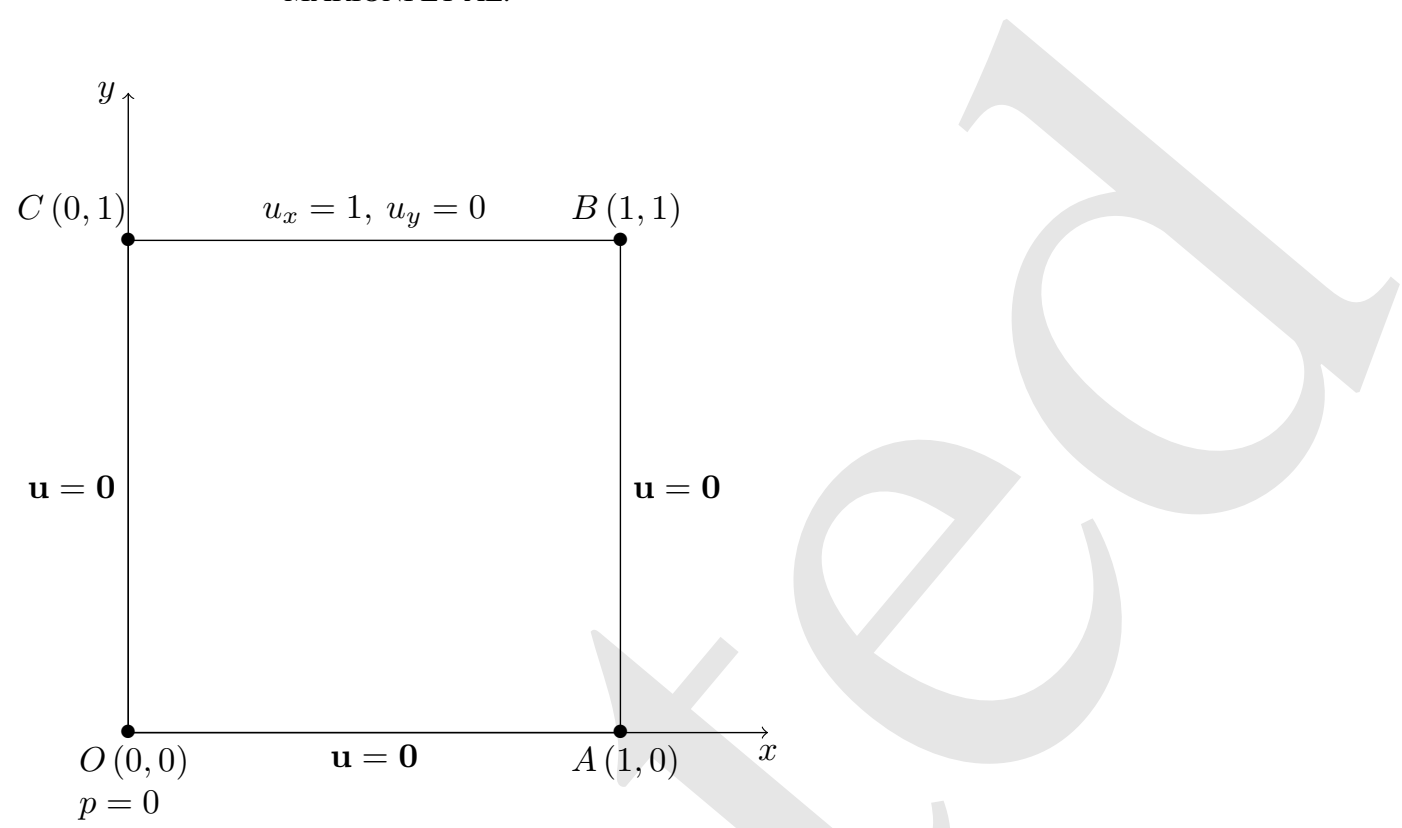

Figure 1. Schematic representation of the lid-driven cavity test: geometrical features and boundary conditions

decrease the turbulence. In these kind of applications, the magnetic Reynolds number is usually Rem $<<1$, so the magnetic field convection has a small role in the development of the magnetic field. A given turbulence level can be obtained by imposing a certain magnetic field. The problem is that this level does not depend only on the Stuart number, $N=H a^{2} / R e$. By rewriting $N$ in terms of material parameters we have $N=\left(B^{2} L \sigma\right) /(\rho u)$, so it does not depend on the fluid viscosity. Thus a high Stuart number allows us to brake the flow, but the expected braking will occur at different level of $N$, depending on the material viscosity. For low viscosity fluids, $R e$ will increase, while $N$ will not, so high $B$ will be needed to completely brake the flow. Therefore, we want to study the numerical behaviour of the solution when the imposed magnetic field is high, which induces a fast transient state in the flow. All the simulations in this work have been performed by using $\mathrm{CimLib}^{\circledR}$ which is a set of libraries developed in the Lab and used as base for the commercial software TherCast ${ }^{\circledR}$.

\section{MATHEMATICAL MODEL}

The considered test case has been sketched in figure 1. The 2D square cavity has length $L=1$; no-slip boundary conditions have been imposed at three borders while a constant tangent unity velocity has been imposed at the last border. The zero level pressure has been imposed to the vertex opposite to the applied shear velocity. The gravity is set to zero and the magnetic field is imposed in different configurations.

A newtonian, incompressible and conductive fluid has been considered. The density has been set to $\rho=10^{3} \mathrm{~kg} / \mathrm{m}^{3}$ and the dynamic viscosity $\mu$ has been set in accordance to the target Reynolds number $R e=\frac{\rho u L}{\mu}$. The electric conductivity is set to $\sigma=7.14 \times 10^{5} \Omega \mathrm{m}$. The external magnetic field $\left(B_{0}\right)$ is constant; the Reynolds magnetic number is assumed to be less than one, hereby the induced magnetic field produced by the conductor's motion is neglected. Thus, the interaction between the electromagnetic fields (EMF) and the flow consists in the Lorentz force:

$$
\mathbf{F}_{\mathbf{L}}=\mathbf{j} \times \mathbf{B}_{\mathbf{0}}
$$


with $\mathbf{j}$ to be the electric current density computed according to the Ohm's law:

$$
\mathbf{j}=\sigma\left(-\nabla \phi+\mathbf{u} \times \mathbf{B}_{\mathbf{0}}\right)
$$

where $\phi$ is the electric scalar potential. The potential has to respect the current density conservation condition

$$
\begin{aligned}
& \nabla \cdot \mathbf{j}=0 \\
& \Delta \phi=\nabla \cdot\left(\mathbf{u} \times \mathbf{B}_{\mathbf{0}}\right)
\end{aligned}
$$

which is naturally satisfied for any in-plane magnetic field vector superimposed to a $2 \mathrm{D}$ flow, since

$$
\Delta \phi=\partial_{z}\left(u_{x} B_{y}-u_{y} B_{x}\right) \equiv 0
$$

The Lorentz force is added in Navier-Stokes equations as a volumetric force term:

$$
\left\{\begin{array}{l}
\rho(\partial \mathbf{u}+\mathbf{u} \cdot \nabla \mathbf{u})-\nabla \cdot(2 \mu \varepsilon(\mathbf{u})-p \mathbf{I})=\mathbf{F}_{L} \\
\nabla \cdot \mathbf{u}=0
\end{array}\right.
$$

The equations are solved by a variational multiscale approach in the framework of stabilized finiteelements. For the detailed analysis of the method, we recall to [8]. In this work we limit to notice that the electromagnetic force is tracked explicitly in time, so it affects the Galerkin resolution and the stabilization terms as a known source term.

\subsection{Time-advancing scheme}

In this work we use the classical $\theta$-scheme for the variational multi-scale (VMS) algorithm used in [8]. The main difference is the presence of an additional term, i.e. the Lorentz force; this term is considered as an explicit source term [22], thus it is evaluated at the $(n-1)$ time step and considered constant through the step. This means that, under the hypothesis of a globally braking effect of the magnetic field, we overestimate the force due to the linearization inside the time step. The aforementioned overestimation is not a problem for low level of the magnetic interaction parameter: in this case the transient flow induced by the magnetic field has a characteristic time scale much higher than the time step, classically computed according to the $C F L$ condition:

$$
\Delta t<\frac{C \Delta x}{u}
$$

where $u$ is the fluid velocity, $C$ is a constant and $\Delta x$ is the characteristic dimension of the element. For the current work we have $\Delta x \simeq 0.01, u=1$ and $C>1$, since $C=1$ is the usual limit for explicit time schemes. In [8] we showed that for the current problem $\Delta t=0.1$ can be used.

Different problems arise when the magnetic interaction parameter is increased. For high $N$, the transient flow's time scale could be of the same magnitude as the $\Delta t_{C F L}$ : in this configuration a different $\Delta t$ has to be calculated to both obtain accuracy and convergence. To do this, we consider a purely inertial flow, which is reasonable at high $R e$. This is also justified in respect to continuous casting, where the metal density is high as well as the turbulence. We want to find the time-step by which the piece-wise constant Lorentz force completely brakes the flow in one time-step. So we compare the work of the external force to the internal energy, approximated to the kinematic energy:

$$
\int d \mathcal{E}=\int \delta \mathcal{W} .
$$

We consider the force to be constant in time and opposite to the velocity, thus:

$$
\frac{1}{2} \rho u_{0}^{2}=f_{L} s(t) .
$$


where $s(t)$ is the displacement of a material particle during the time step.

By considering a material particle far enough from the boundary, and by assuming the magnetic field normal to the velocity (most restrictive case), we can reduce the motion of the particle to a constant decelerating motion

$$
s=u t+\frac{1}{2} a t^{2}=u t-\frac{1}{2} \frac{f_{L}}{\rho} t^{2} .
$$

By substituting equations (1) and (9), we can rewrite equation (8) as:

$$
\begin{gathered}
-\frac{1}{2 \rho}\left(\sigma B^{2}\right)^{2} \Delta t^{2}+\sigma B^{2} \Delta t-\frac{1}{2} \rho=0 \\
\Delta t^{*}=\rho \frac{1}{\sigma B^{2}}
\end{gathered}
$$

where $\Delta t^{*}$ is the threshold where the work of the external force is high enough to dissipate all the kinetic energy in one time-step. Similarly, $\Delta t=2 \Delta t^{*}$ will be the time step which will allow the force to induce a flow opposite to the flow at the previous time-step in the areas far enough from the boundary.

\subsection{Anisotropic mesh adaptation}

In [23] a posteriori error estimate based on the length distribution tensor approach and the associated edge based error analysis is proposed. This error estimation algorithm is the base for the anisotropic meshing scheme adopted in the current work. In this section we propose a synthesis of the method based on [23] and further developed in [9] in the case of Navier-Stokes, multi-field re-meshing.

Let us introduce the set of fields we want to remesh according to:

$$
\mathbf{u}=\left\{u_{1}, u_{2}, \ldots, u_{N}\right\} .
$$

Let's than denote the error of each field along the axis as:

$$
\mathbf{e}_{i j}=\left\{e_{i j}^{1}, e_{i j}^{2}, \ldots, e_{i j}^{N}\right\}
$$

which can be expressed as:

$$
e_{i j}^{n}=\mathbf{g}^{i j} \cdot \mathbf{X}^{i j}
$$

where $\mathbf{X}^{i j}$ is the length of the edge linking the $i-t h$ node to the $j-t h$ node, and $\mathbf{g}^{i j}=\mathbb{H}(u) \mathbf{X}^{i j}$. Thus, we can define the error along the edges as

$$
e_{i j}^{n}=\mathbf{g}^{i j} \cdot \mathbf{X}^{i j} .
$$

Now we want to consider the error in relation to a change in the edges' length, express in term of stretching factors $s \in \mathbb{R}$, so that:

$$
\left\{\begin{array}{l}
\tilde{\mathbf{X}}^{i j}=s_{i j} \mathbf{X}^{i j} \\
\tilde{e}_{i j}^{n}=s_{i j}^{2} \mathcal{G} \cdot \mathbf{x}^{i j}
\end{array}\right.
$$

with $\tilde{\mathbf{X}}^{i j}$ and $\tilde{e}_{i j}^{n}$ being the target edge length and the target error respectively and $\mathcal{G}$ a recoverygradient operator defined in [23].

Let us define the set of stretching coefficients as

$$
\mathcal{S}=\left\{s_{i j} \in \mathbb{R}^{+} i=1, \ldots, \Lambda, j=1, \ldots, \Lambda, \Gamma(i) \cap \Gamma(j) \neq \emptyset\right\}
$$

with $\Lambda$ being the number of nodes of the mesh; we can therefore derive the associate metric $\mathbb{M}$ as:

$$
\mathbb{M}^{i}=\left(\frac{1}{d} \sum_{j \in \Gamma(i)} s_{i j}^{2} \mathbf{X}^{i j} \bigotimes \mathbf{X}^{i j}\right)^{-1} .
$$




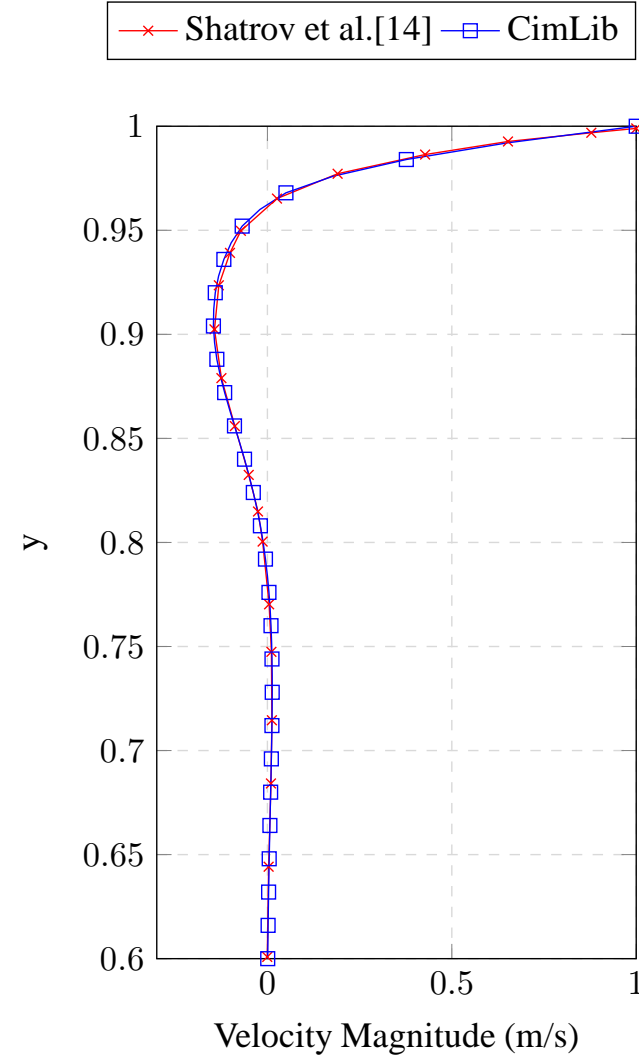

(a) Velocity profile along the middle-line, $R e=5000$, $N=5$
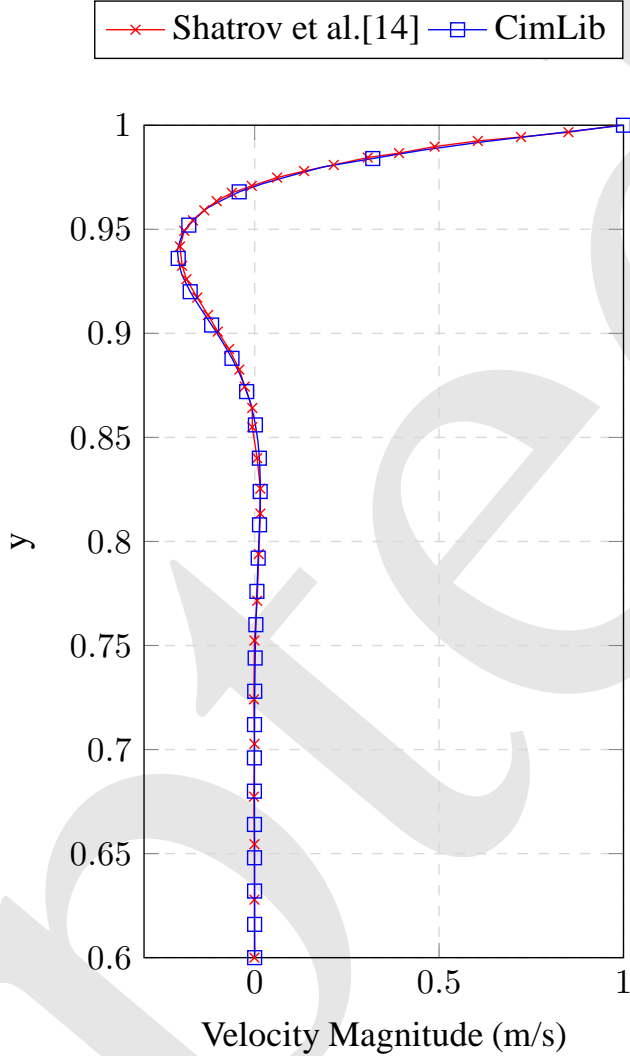

(b) Velocity profile along the middle-line, $R e=5000$, $N=30$

Figure 2. Velocity profile along the middle-line: validation

For the complete derivation of the metric, please refer to [23].

Set the metric framework, we need now to choose the input field to remesh according to. For highReynolds incompressible flows, we propose the following set of fields:

$$
u\left(\mathbf{X}^{i}\right)=\left\{N_{v} \frac{v^{i}}{\left|v^{i}\right|}, N_{s} \frac{\left|v^{i}\right|}{\max _{j}\left|v^{j}\right|}\right\}
$$

where $N_{s}$ and $N_{v}$ are weighting parameters and $v$ is the velocity field

\section{RESULTS}

\subsection{Horizontal magnetic field and validation}

The code in absence of magnetic field has been largely validated in literature [8,9] for the lid-driven case, hence in the current work we limit to validate the case with $B_{0} \neq 0$. For the first test case, we set $B_{0}$ in the same direction as the $\mathrm{x}$ axis. In figure $2, u_{x}$ profile along the vertical middle-line is plotted. The benchmark is taken from [14] and $R e=5000$ and $N=5,30$ are reported in figure 2(a) and 2(b) respectively. In figure 3 the velocity magnitude in the upper part of the cavity is plotted. We can notice that the braking effect increases as $N$ increases and the vertical velocity (normal to $B_{0}$ ) is dumped. This leads to a more horizontal flow and thinner eddies, as shown in figure 4.

Even with higher $R e$ the stabilizing effect of the magnetic field occurs: in figure 5 the evolution of the flow in respect to the magnetic interaction parameter is shown. The number of eddies is directly related to the Stuart number, as reported in figure 6 . Note that in figure 6 a non-continuous 

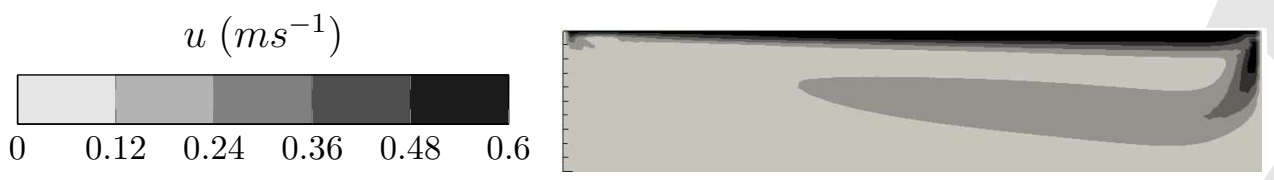

(a) Velocity magnitude in $y \in(0.8 ; 1): R e=5000, N=5$

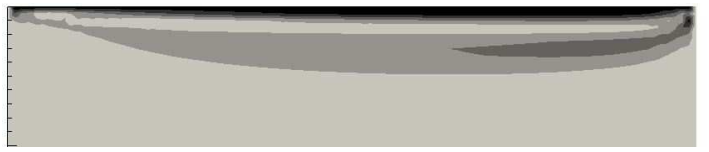

(b) Velocity magnitude in $y \in(0.8 ; 1): R e=5000, N=30$

Figure 3. Velocity magniture in the upper part of the cavity
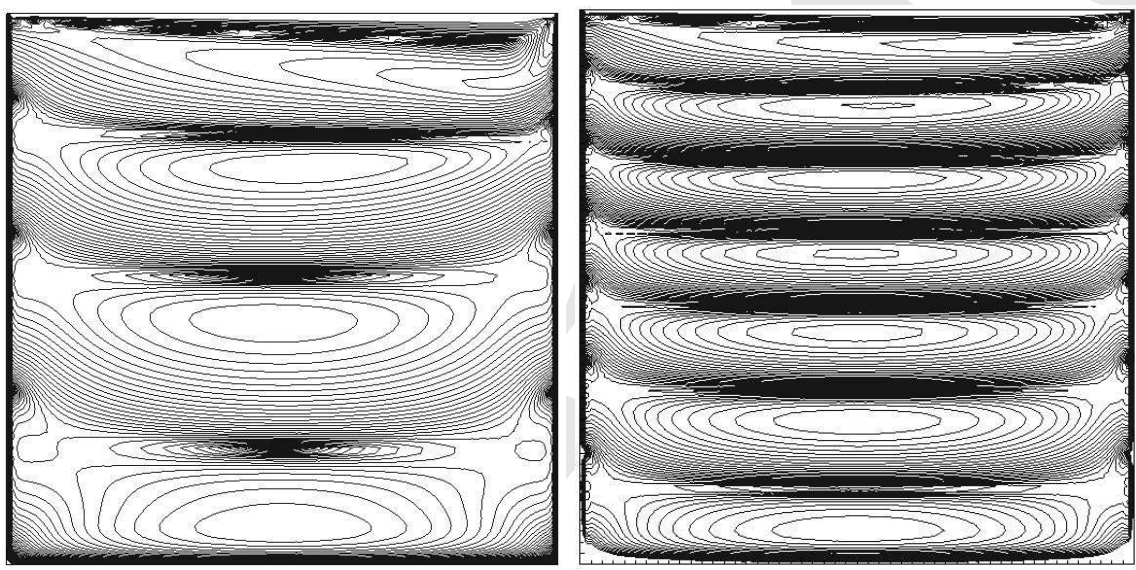

(a) Velocity magnitude isocontour: $R e=5000$, (b) Velocity magnitude isocontour: $R e=5000$, $N=5$ $N=30$

Figure 4. Isocontour of velocity field magnitude. The isovalues are in logarithmic scale: 20 in the range $\|u\| \in(0.01 ; 0.5)$ and 100 in the range $\|u\| \in(0 ; 0.01)$

variable, the number of eddies, is plotted so the linear function represents the tendency of a piecewise constant function. As we see from figure 5, the different $N$ adopted do not represent the last (and most unstable) eddy at the same level of development. The increasing of $N$ is a constrain over the vertical flow which leads to almost horizontal re-circulation patterns. In figure 7 the average slope of the interface between the two upper eddies is plotted; by increasing $N$ an alternating horizontal flow is induced and the vertical re-circulation is confined at the external boundaries. In figure 8 the horizontal velocity along the vertical middle-line is plotted. By increasing the magnetic field, the main horizontal flow moves upwards and the thickness is almost constant. At high $N$, the eddy is pushed against the lid, its thickness decreases and its velocity increases. Note also that the horizontal velocity of the secondary eddies is much lower than the velocity of the first upper eddy. As the eddies' thickness decreases with the increasing of $B$, remesh is fundamental to catch the small eddies without increasing the number of elements. In figure 9 we see how the mesh anisotropically adapts to the flow. From figure 9(c) it is possible to notice that the algorithms does not catch the low velocity eddies by itself. For this reason, the weighting parameter $N_{s}$ and $N_{n}$ are computed according to the magnetic interaction parameter. The anisotropic non-structured mesh allows the user to well model the boundary layers and the area close to the corners, where instabilities due to the boundary conditions non continuity may occur. 


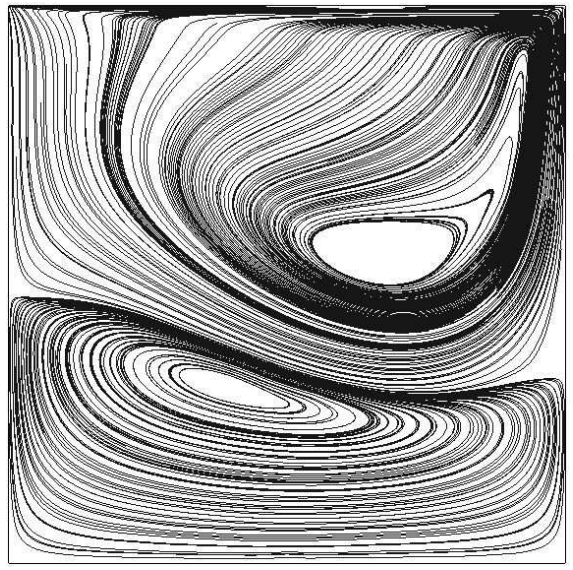

(a) Velocity streamlines: $R e=10000, N=0.5$

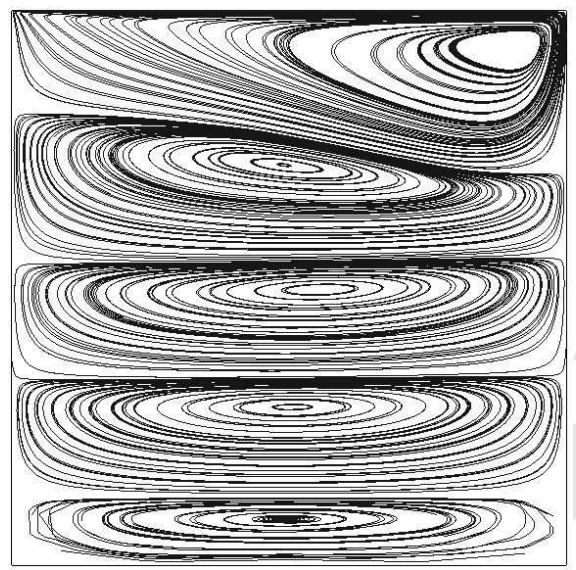

(c) Velocity streamlines: $R e=10000, N=2$

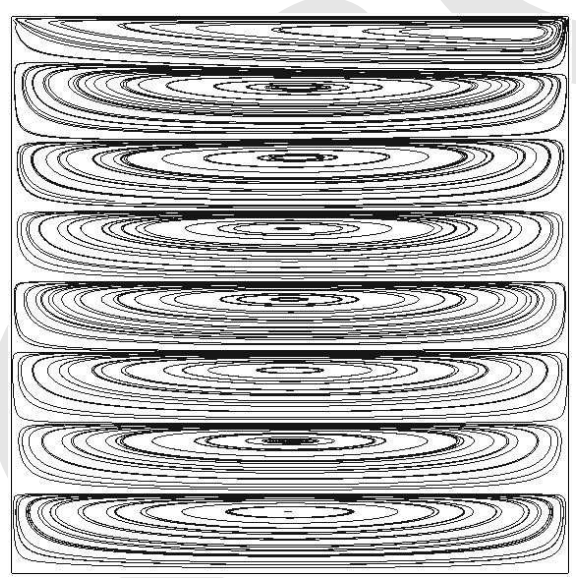

(e) Velocity streamlines: $R e=10000, N=15$

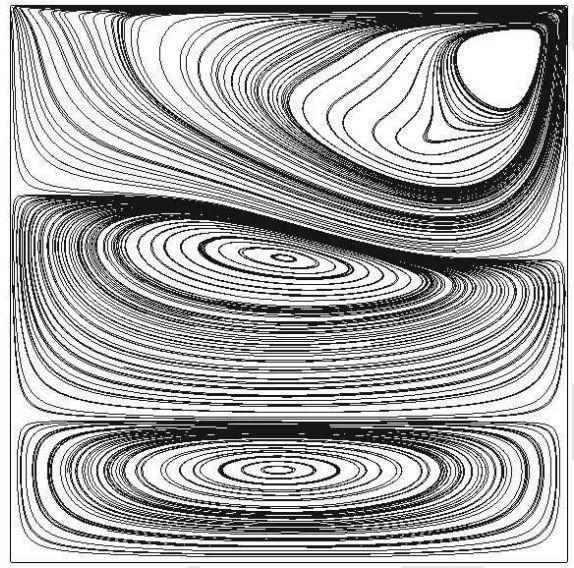

(b) Velocity streamlines: $R e=10000, N=1$

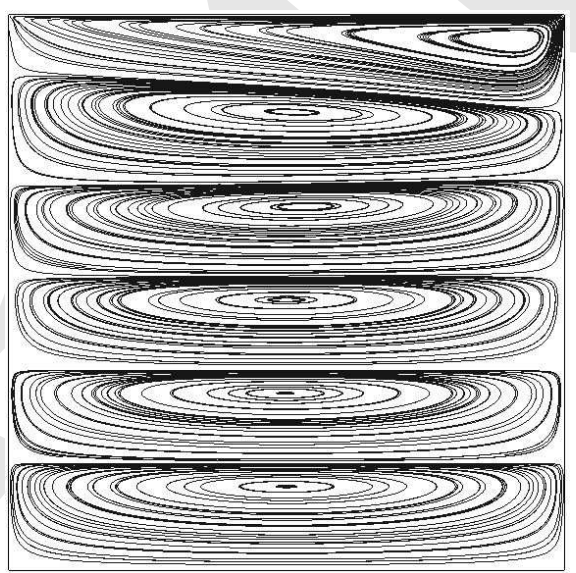

(d) Velocity streamlines: $R e=10000, N=5$

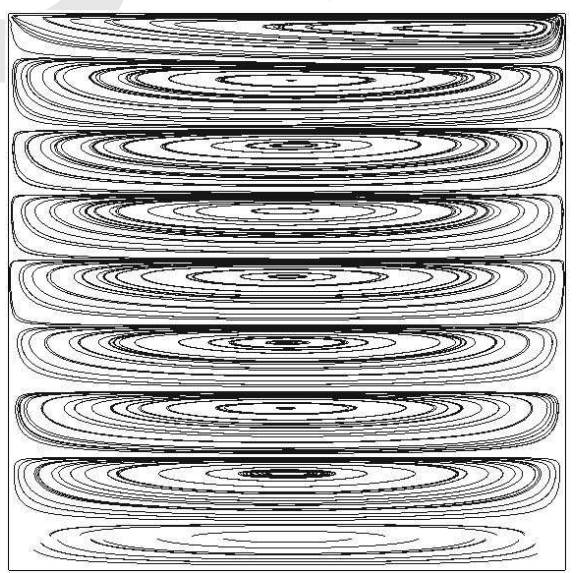

(f) Velocity streamlines: $R e=10000, N=20$

Figure 5. Velocity streamlines of the flow subjected at different external magnetic field's magnitudes

\subsection{Vertical magnetic field}

In the lid-driven cavity test, the horizontal flow is the primary one, since it's forced by the boundary condition. An horizontal magnetic field opposes to the vertical circulation of the flow, stimulating 

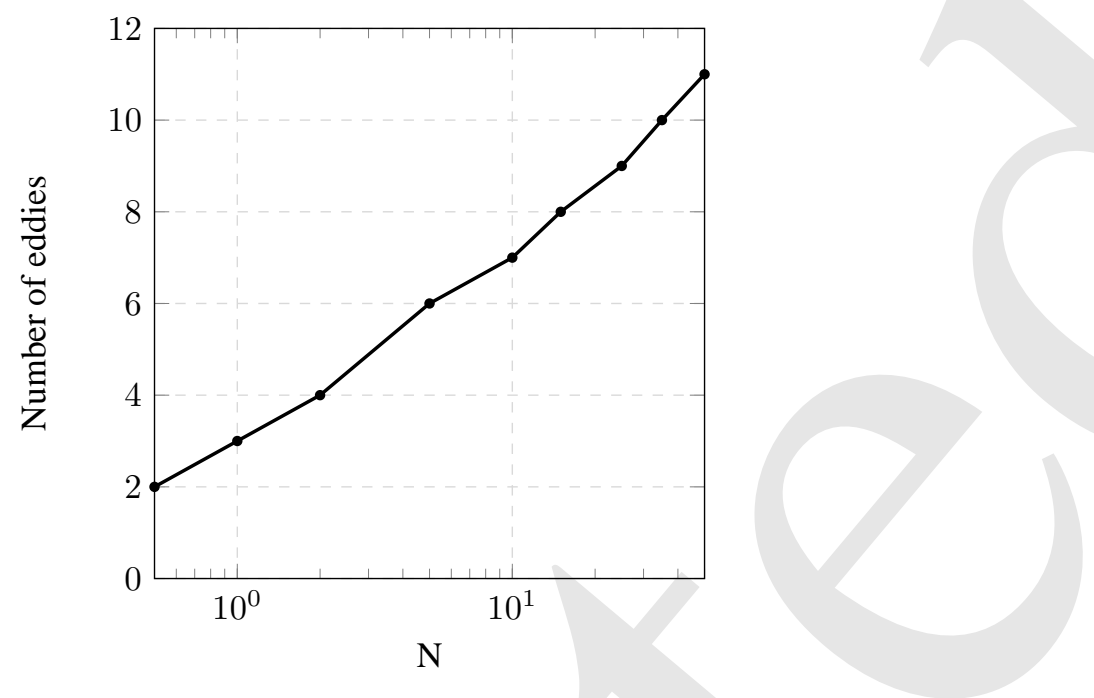

Figure 6. Number of horizontal eddies with respect to the magnetic interaction parameter

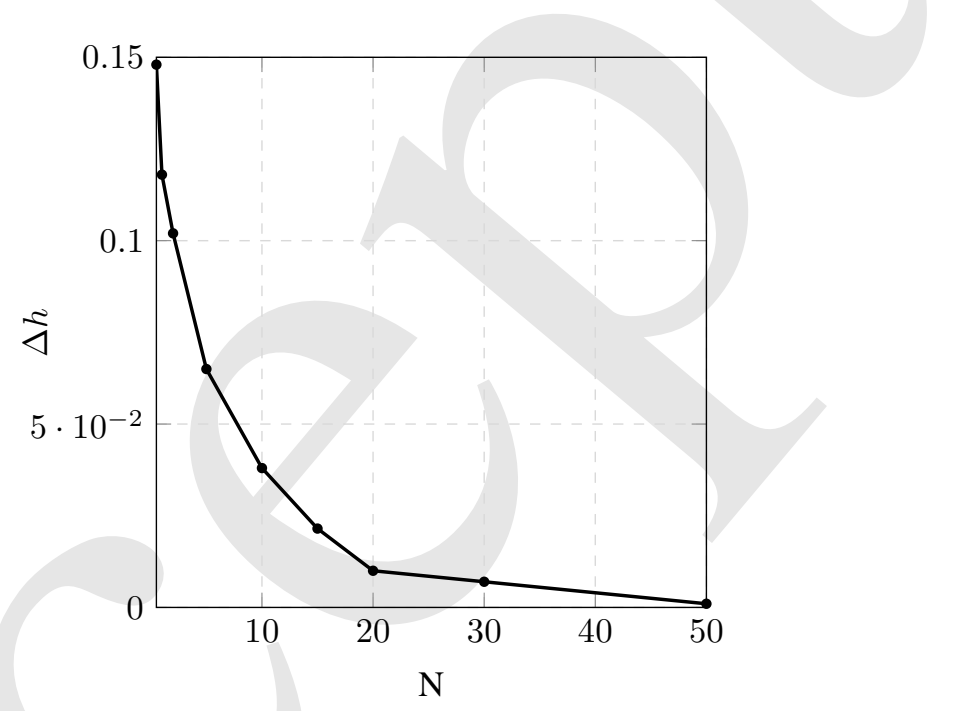

Figure 7. Average slope of the interface between the two upper eddies

the horizontal flow. In this way we decrease the importance of the secondary vertical flow, so we create different eddies almost independent; the upper b.c. induces a first eddy, whose bottom part induces the second eddy below and so on. In order to fully interact with the mechanical behaviour we decided to consider a magnetic field facing the primary flow. In the second test case the orientation of the magnetic field was changed, so that $B_{y}=$ const. and $B_{x}=B_{z}=0$. In this configuration the primary flow, driven by b.c., must cross the magnetic field, so a higher level of braking is expected. In figure 10 the flow at different Stuart numbers is described. The first effect of the magnetic field is to sharpen the flow at the upper-right corner and to suppress the smallest eddies (figure 10(b)). By increasing the Stuart number the main eddy squeezes along the right boundary, where the kinetic energy input by the b.c. is highest. Finally, in figure 10(f), the flow is controlled by the magnetic field. The fluid coming from the upper boundary deviates in the $y$ direction since the magnetic field obstacles the horizontal flow. Despite this, the flow has to move to the left side because of the boundary conditions on velocity and so it can not form an independent eddy. For this reason a slow, laminar flow is formed in the middle of the cavity. Over this value of $N$, the vertical eddy's size would decrease as well as the magnitude of velocity in the center band. The height of 


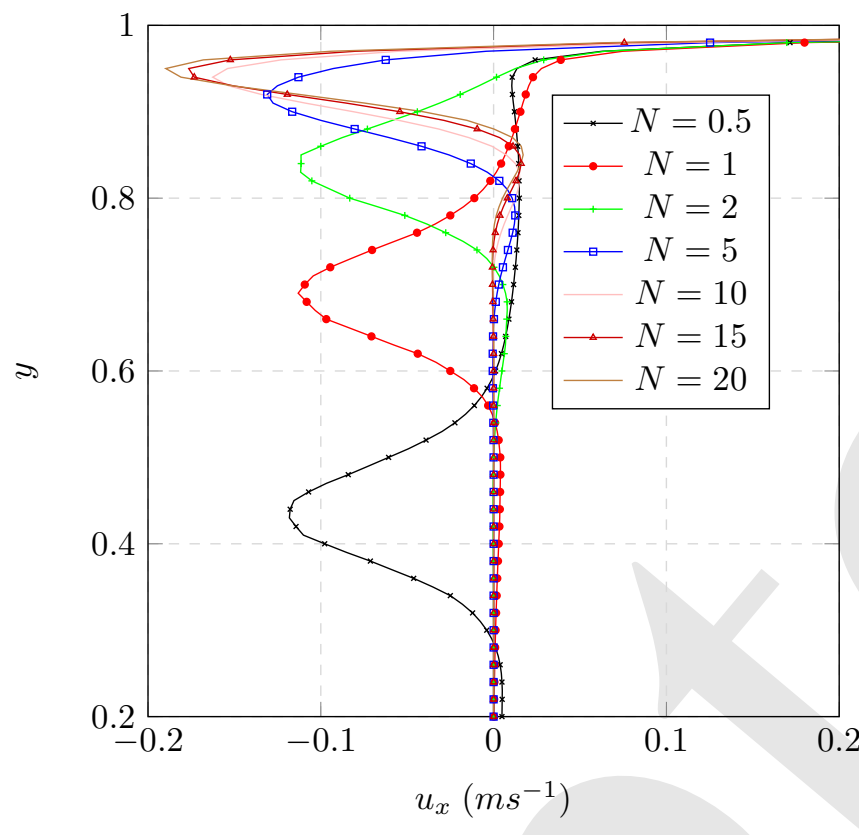

Figure 8 . Horizontal velocity profile along the vertical middle-line

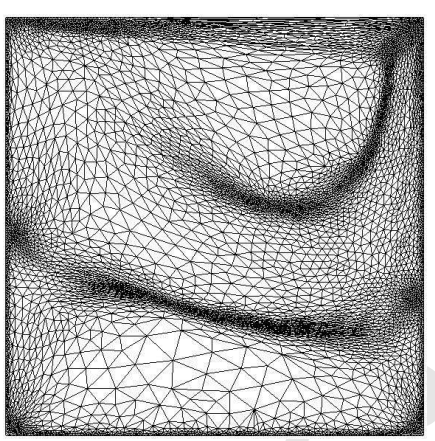

(a) Final mesh: $R e=10000, N=$ 0.5
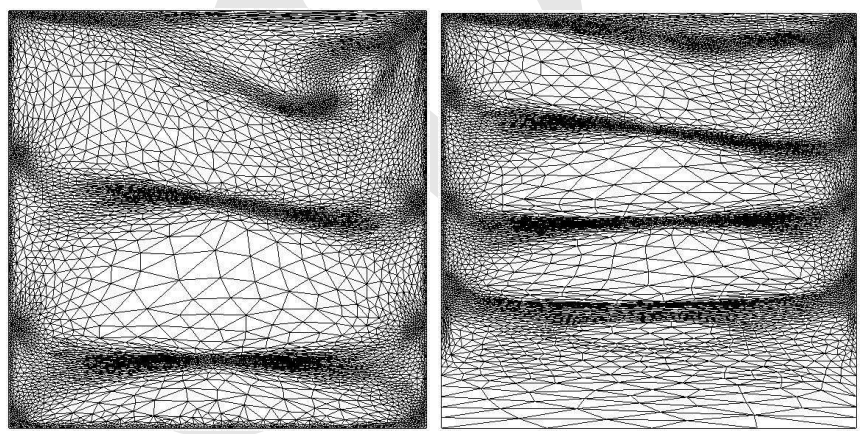

(b) Final mesh: $R e=10000, N=(\mathrm{c})$ Final mesh: $R e=10000, N=$

Figure 9. Dinamically adapted anisotropic mesh

the right eddy depends on the Reynolds number: as reported in table I a higher Reynolds number leads to a higher vertical excursion of the eddy. It is also shown a very known result, by which a high Stuart number can regularize the turbulent flow and make it steady. In figure 11 the horizontal velocities along the horizontal middle-line are reported. We notice that the constant velocity band in the middle is wider when $R e$ is higher (and $\mu$ lower); the low-viscosity flow is therefore constrained into a thin and high eddy close to the right boundary. The same process is clear from the same plot for a higher Stuart number (figure 12). The curve for the same $R e(R e=10000$ and $R e=20000$ ) are more braked, thus the velocity picks decrease and the plateau is wider (until almost $x=0.8$ against about $x=0.55$ ).

In figure 13 we plot the number of nodes where the velocity normal to the magnetic fields reverses over one time step, i.e. $u^{n} \cdot f^{n}>0$; in this case the Lorentz force brakes the flow and then accelerates it inside the same time increment. Since the Lorentz force does not follow the velocity inside the time step, it happens that for fast transient phenomena the $\Delta t_{C F L}$ is of the same magnitude than the transient flow itself. From figure 13 we see that $\Delta t=3 \cdot 10^{-3} \mathrm{sec}$ leads to invert 


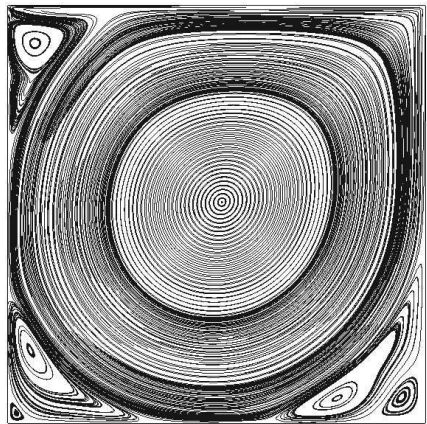

(a) Velocity $R e=10000, N=0$

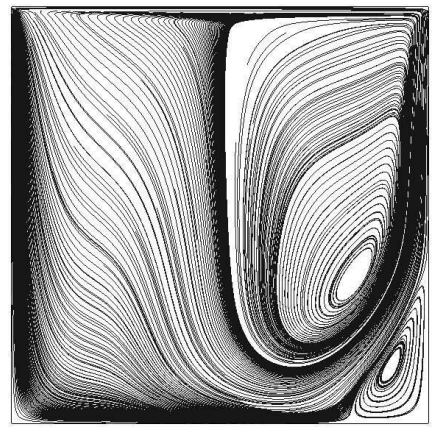

(d) Velocity streamlines, (e)

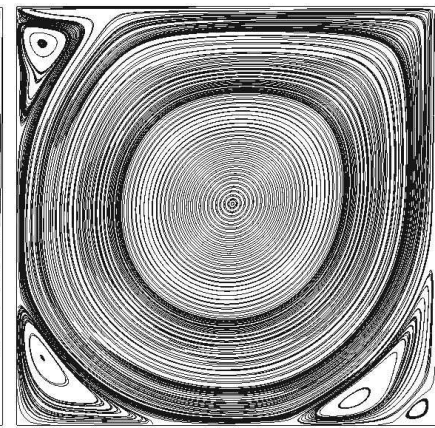

streamlines, (c) $R e=10000, N=0.01785$

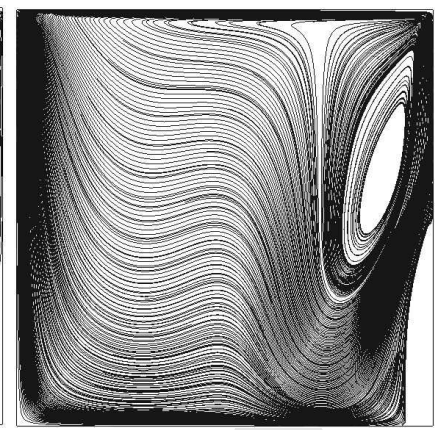

(e) Velocity $R e=10000, N=1.785$

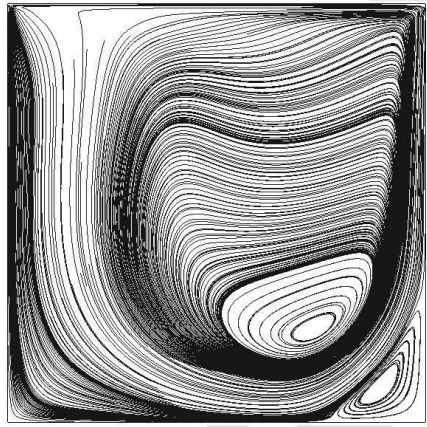

streamlines, $R e=10000, N=0.2856$

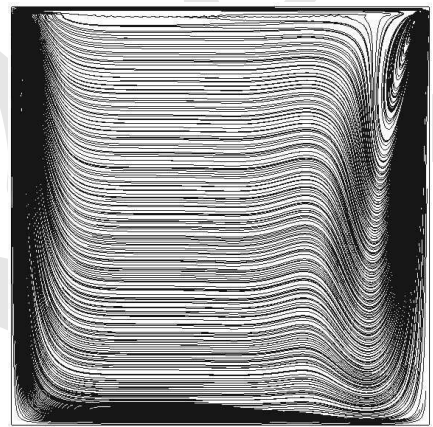

(f) Velocity streamlines, $R e=10000, N=7.14$

Figure 10. Velocity streamlines of the lid-driven cavity flow under a vertical magnetic field.

Table I. Minimum height of $\|\mathbf{u}\|=0.05$ isovalue

\begin{tabular}{c|c|c}
$R e$ & $y_{N=7.14}$ & $y_{N=1.785}$ \\
\hline 200000 & 0.11 & non-steady \\
100000 & 0.165 & non-steady \\
50000 & 0.22 & 0.09 \\
20000 & 0.33 & 0.148 \\
10000 & 0.44 & 0.175 \\
5000 & 0.5 & 0.21 \\
1000 & 0.52 & 0.258
\end{tabular}

the flow in the $55 \%$ of the domain. It is important to underline that in a Eulerian framework, this is a natural phenomenon: the velocity variation is referred to the spatial deformation of the flow and not on the material particle itself. In this case the pathological behaviour is evident from the fast increase of the number of these points, as clear from figure 13. To better analyse this problem, we will consider the most restrictive case in section 3.3.

\subsection{Perfect braking case}

In this section we will present a numerical test in order to better catch the numerical problem shown in figure 13. We propose to consider a Lorentz force opposite to velocity on the whole domain (perfectly braking) and equal to:

$$
f_{L}=\sigma u B^{2}
$$

By this test we will be able to: 


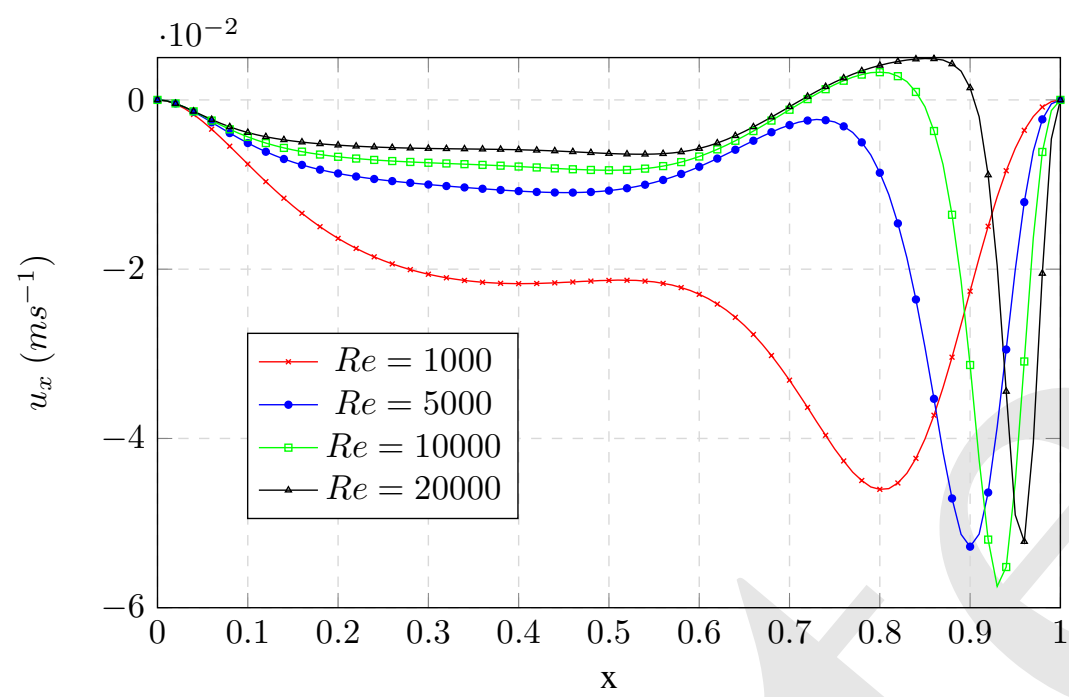

Figure 11. $u_{x}$ plot over the center-line $y=0.5$ at different $R e$ and $N=1.785$

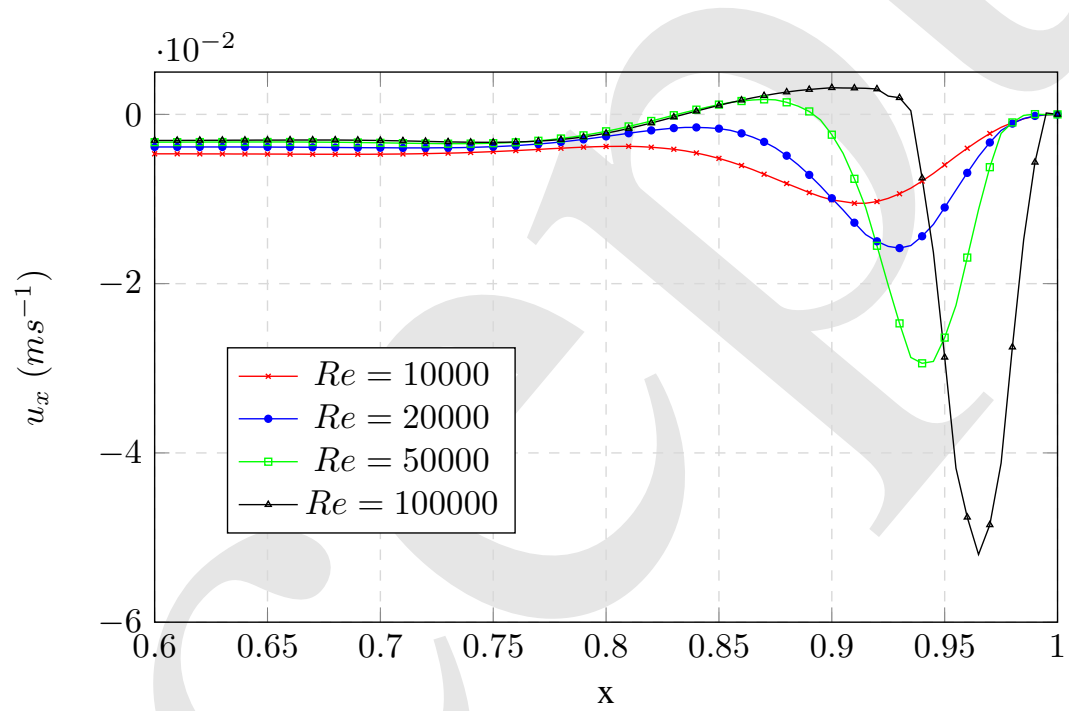

Figure 12. $u_{x}$ plot over the center-line $y=0.5$ at different $R e$ and $N=7.14$

- Minimize the flow deformation. As stated in section 3.2, "reverse velocity" point could be naturally produced by the flow deformation in Eulerian simulations. By imposing a perfectly braking force, the flow will be slowed but not heavily deformed. The pathological velocity inversion will occur on the whole domain for the same time step, so it will be easily detected.

- Extend the numerical problem on the whole domain. In the cases presented in sections 3.1 and 3.2 , the numerical problems occurred in the regions where magnetic reaction to the flow was maximum, so where the velocity was normal to the magnetic field. By imposing a perfectly braking force we will be able to extend the same conditions to the whole domain.

This numerical test case represents all the physical cases in which the Lorentz force is opposite to the velocity globally (e.g. vertical flow with an horizontal magnetic field) or locally (e.g. cases in sections 3.1 and 3.2).

Several tests similar to the one reported in figure 13 have been conducted and the results are reported in figure 14. In all these configurations we notice that the turn of velocity is much faster 


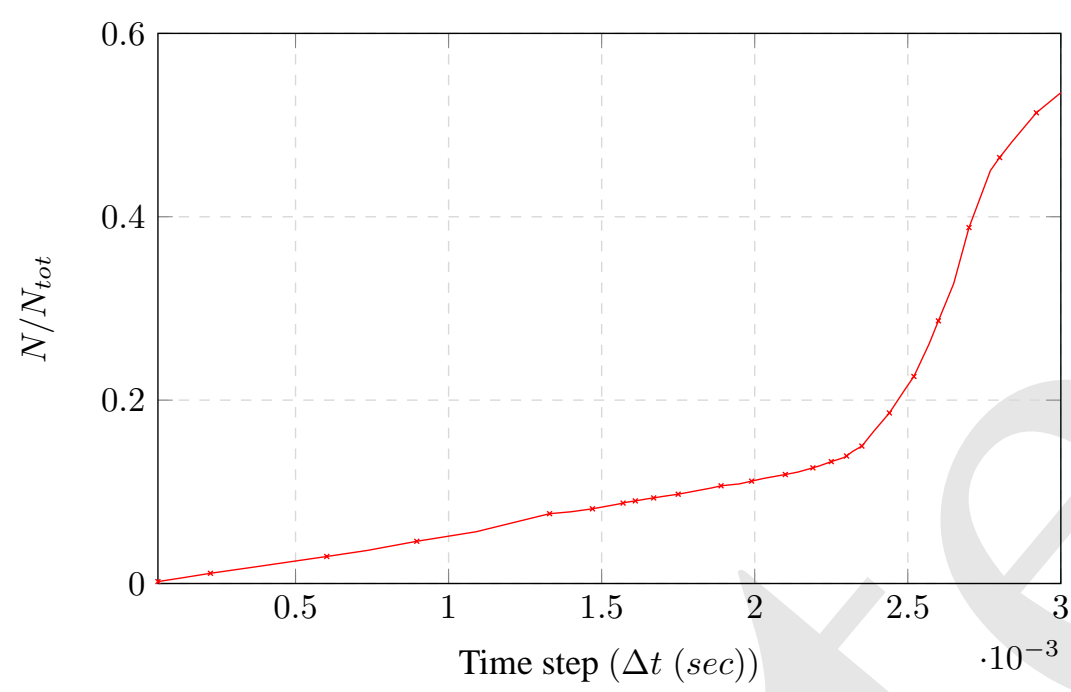

Figure 13. Number of reverse-velocity nodes normalized to the total number of free nodes, $N=7.14$

than in the case of vertical magnetic field. In figure 13 the velocity turning is smoother because part of the Lorentz force's work is spent to deform the flow; in this case the extra-constraint on deformation leads to an almost instantaneous velocity turning, where the energy dissipated inside the time step is high enough to balance the inertia. We can also notice that the expression (10) well predicts the time-step which leads to this phenomenon, represented by the dotted lines (notice that few different time steps were used for each simulation, so the whole band is included between the smaller and larger time steps used). We do not report the complete curves for all the configurations analyzed, but in figure 15 we show the variation of the critical time step over the variation of the main problem variables: from this figure we see the the dependencies are conform to the one adopted in (10).

The choice of $\Delta t$ affects also the convergence. In figure 16 the convergence of the $L^{2}$ error is plotted; the error is here computed in respect to an overkilled solution with $\Delta t \ll \min (\Delta t)$. From this plot we see that for $\Delta t \geq 2 \Delta t^{*}$ the simulation diverges, even if we respect the $C F L$ condition. In this case the Lorentz force (constant inside the time-step) is high enough to reverse the flow and increase the velocity magnitude in the opposite direction, which leads to a diverging acceleration of the flow, thus we have

$$
\left\{\begin{array}{l}
u^{n} \cdot u^{n-1}<0 \quad \vee \quad\left\|u^{n}\right\| \geq\left\|u^{n-1}\right\| \\
f_{L}^{n} \cdot u^{n}>0 \quad \vee \quad\left\|f_{L}^{n}\right\| \geq\left\|f_{L}^{n-1}\right\| .
\end{array}\right.
$$

For $\Delta t \in\left(\Delta t^{*} ; 2 \Delta t^{*}\right)$ the solution converges, but the convergence rate is small. In this interval, the transient flow is not well predicted since the punctual velocity reverses its direction at each time-step. The flow will oscillate across an equilibrium point but the reverse velocity's magnitude is still lower than the one at the previous time step, so the oscillations decrease amplitude. This configuration is defined as:

$$
\left\{\begin{array}{l}
u^{n} \cdot u^{n-1} \leq 0 \quad \vee \quad\left\|u^{n}\right\|<\left\|u^{n-1}\right\| \\
f_{L}^{n} \cdot u^{n} \geq 0 \quad \vee \quad\left\|f_{L}^{n}\right\|<\left\|f_{L}^{n-1}\right\| .
\end{array}\right.
$$

For $\Delta t<\Delta t^{*}$ the converge rate is higher and no verse-changing takes place and the following conditions are satisfied:

$$
\left\{\begin{array}{l}
u^{n} \cdot u^{n-1}>0 \quad \vee \quad\left\|u^{n}\right\|<\left\|u^{n-1}\right\| \\
f_{L}^{n} \cdot u^{n}<0 \quad \vee \quad\left\|f_{L}^{n}\right\|<\left\|f_{L}^{n-1}\right\| .
\end{array}\right.
$$




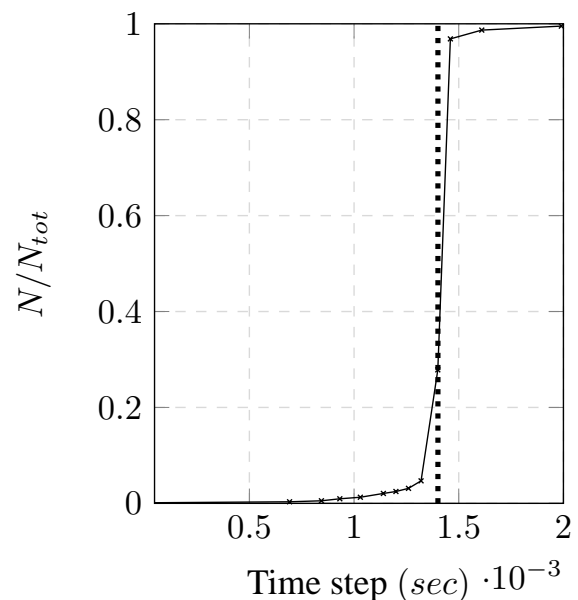

(a) Reverse-velocity points: $\mu=0.1, B_{z}=1$, $\rho=1000, \sigma=714000$

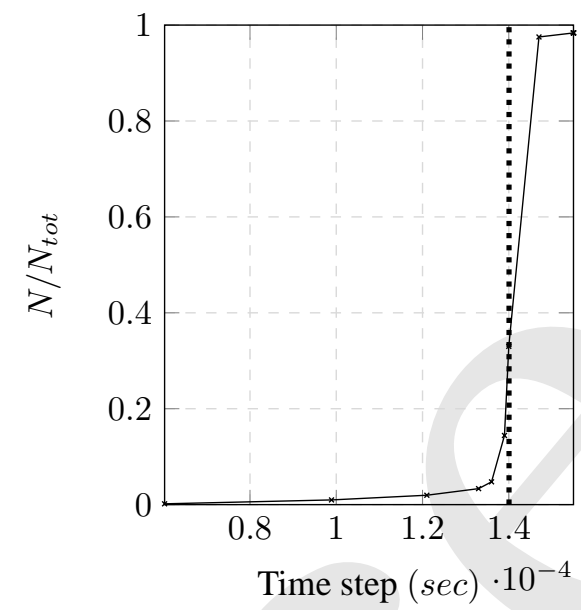

(c) Reverse-velocity points: $\mu=0.1, B_{z}=1$, $\rho=100, \sigma=714000$

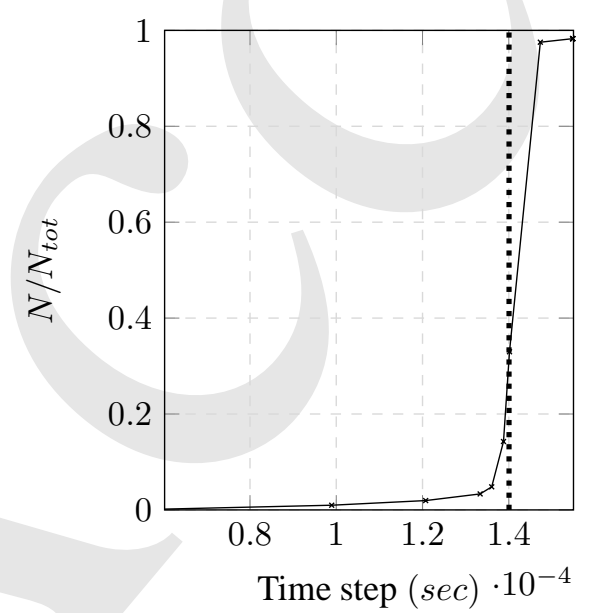

(e) Reverse-velocity points: $\mu=0.1, B_{z}=1$, $\rho=1000, \sigma=7140000$

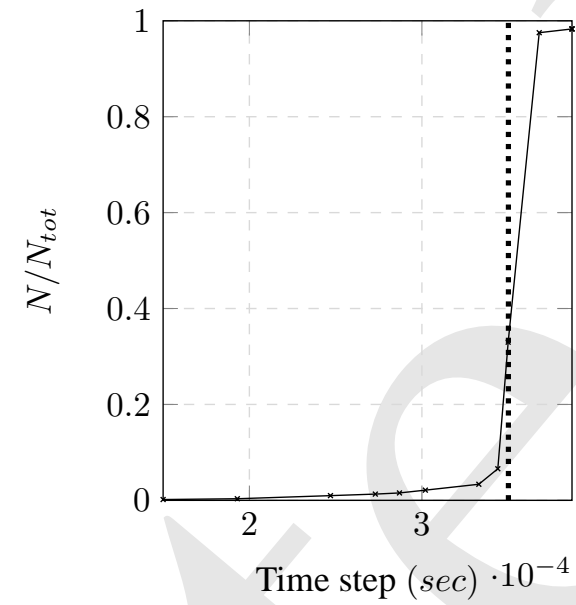

(b) Reverse-velocity points: $\mu=0.1, B_{z}=2$, $\rho=1000, \sigma=714000$

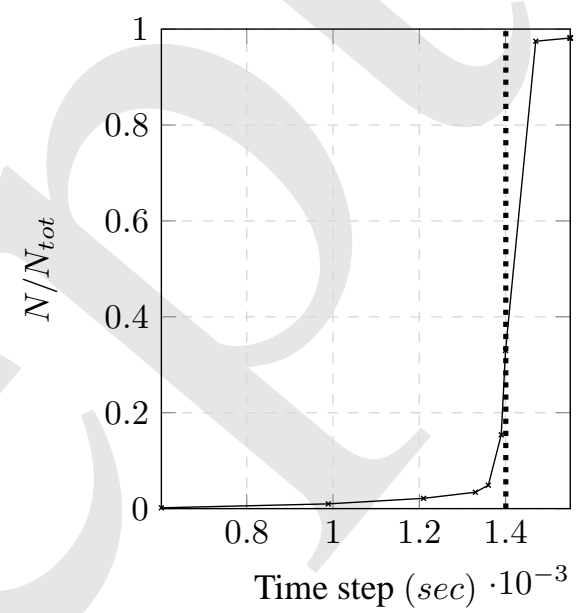

(d) Reverse-velocity points: $\mu=0.1, B_{z}=1$, $\rho=1000, \sigma=714000, u_{\text {b.c. }}=2$

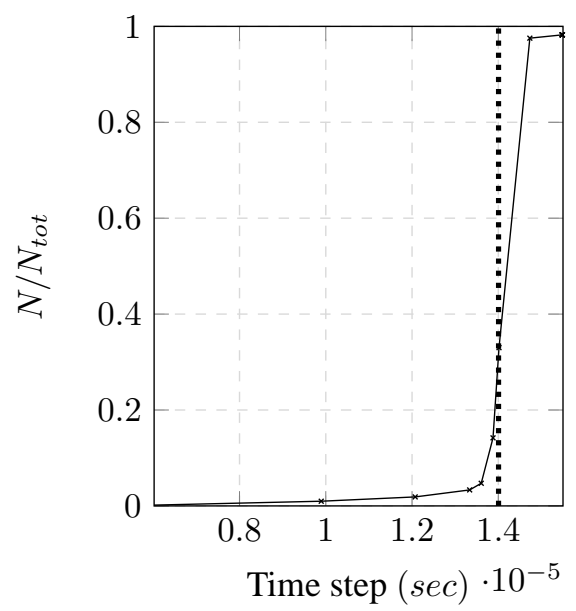

(f) Reverse-velocity points: $\mu=0.1, B_{z}=1$, $\rho=1000, \sigma=71400000$

Figure 14. Normalized reverse-velocity points in different problem configurations 


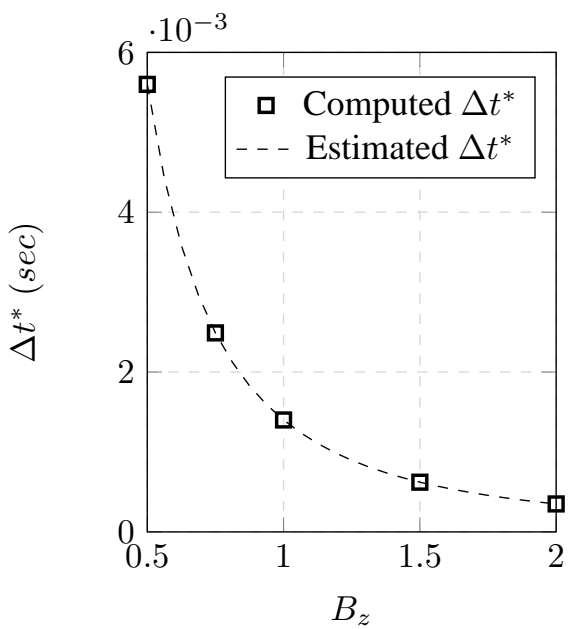

(a) Critical time-step variation at different magnetic field magnitudes

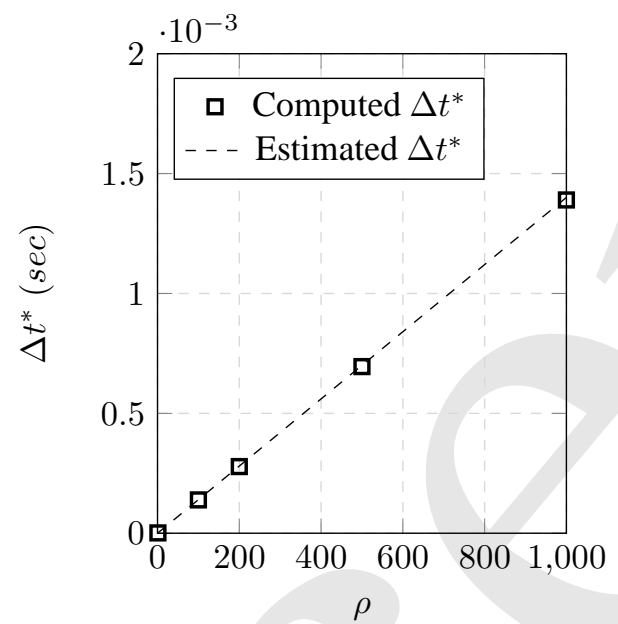

(c) Critical time-step variation at different velocity magnitudes

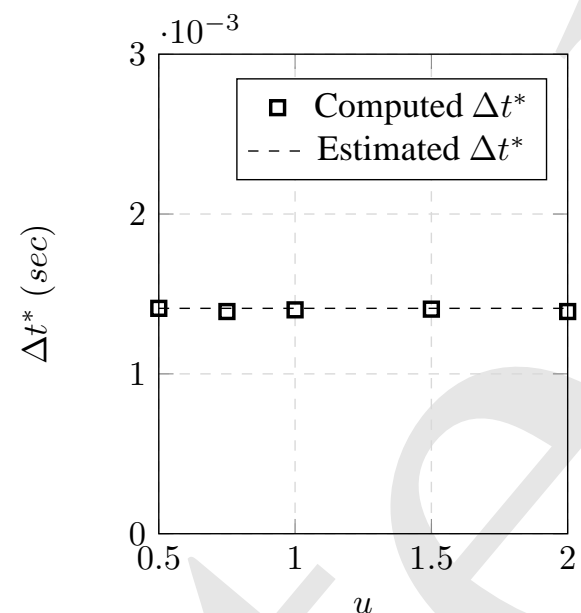

(b) Critical time-step variation at different velocity magnitudes

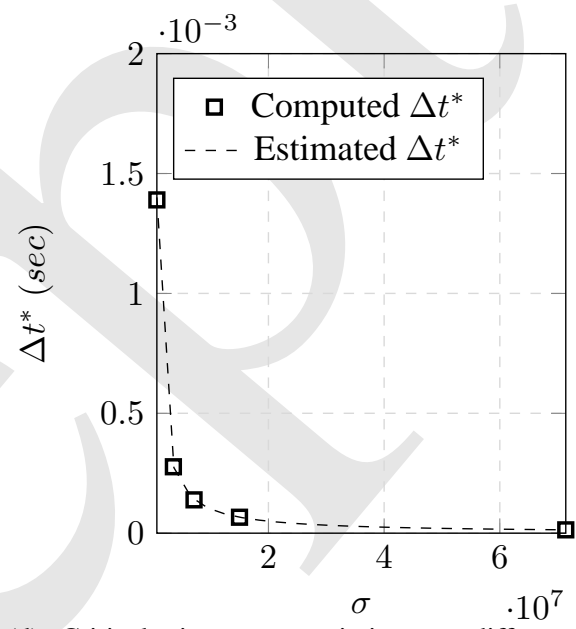

(d) Critical time-step variation at different material's conductivity

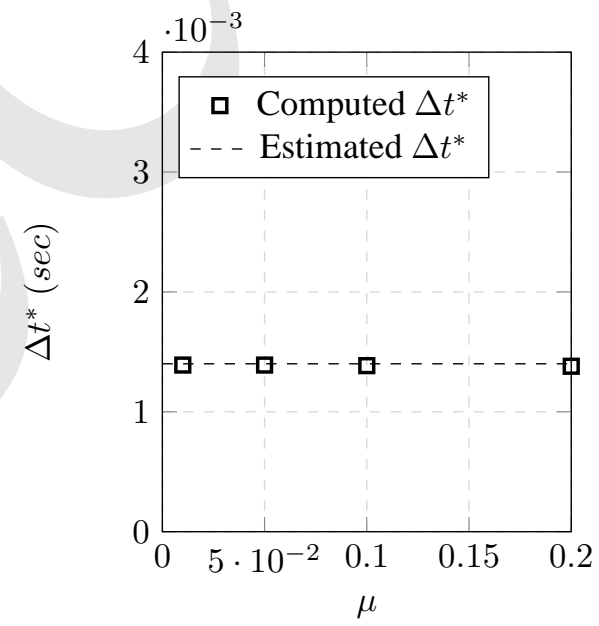

(e) Critical time-step variation at different material's viscosity

Figure 15. Critical time-step variation in respect to main physical parameters' variation 


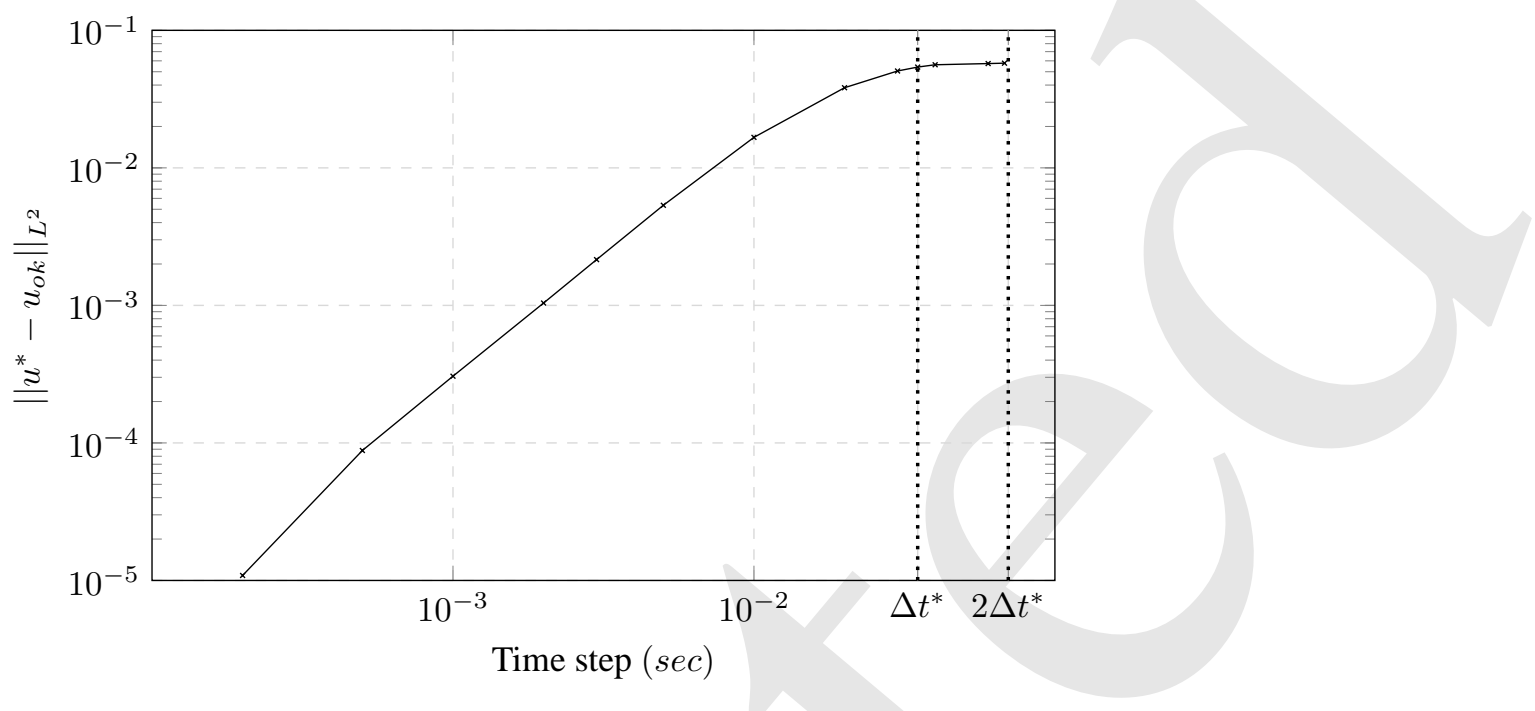

Figure 16. Convergence of error in respect to the refinement in the time discretization

In this case the Lorentz force monotonically brakes down the flow. The velocity's evolution is qualitatively convex and monotonically decreasing, so the piece-wise constant approximation of the Lorentz force leads to an over-estimation of the braking effect, which still does not affect the convergence. These considerations are directly connected to the prediction of the transient state; in figure 17, the time required to reach the steady state is plotted. For $\Delta t=2 \Delta t^{*}$ the required time tends to infinity because the system oscillates between two opposite velocity fields. For $\Delta t<\Delta t^{*}$ the solution tends to a constant value, $T_{s}=0.52$ in the current simulation.

In figure 18 the horizontal velocity at $P(0.5 ; 0.75)$ is plotted over the time-steps. As expected, we see that the simulation with $\Delta t=0.0072>2 \Delta t^{*}$ diverges and the one with $\Delta t=0.0001<\Delta t^{*}$ converges providing a good description of the transient state. Between these cases, we see that for $\Delta t \in\left(\Delta t^{*} ; 2 \Delta t^{*}\right)$ the simulations converge to the correct steady state, but the transient state is oscillatory, due to the numerical problems connected to the time-step. It is also important to note that the velocity, in those cases, does not reverse at each time step forever (like for $\left.\Delta t=0.0072>2 \Delta t^{*}\right)$ : when the velocity is low enough that the viscous energy is dominant in respect to the kinetic energy, the external work is no more sufficient to dissipate the whole energy and equation (10) is no longer valid. In figure 18 , we see that the simulation with $\Delta t=0.006$ stops oscillating at the $16 t h$ step. For $\Delta t=0.0068$, this point is reached at the $68 t h$ step.

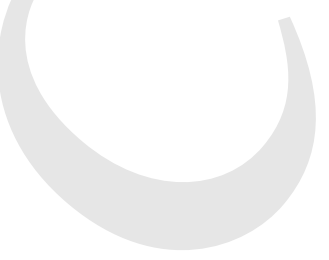

\section{CONCLUSIONS}

In this work high Reynolds driven cavity has been studied. We showed how the flow can be braked and the turbulence decreased by different magnetic field configurations. Indeed, high Reynolds number flows need high magnetic field to be braked, but $\rho$ and $\mu$ play different roles; while $\rho$ affects both $N$ and $R e, \mu$ affects only $R e$, so the $N$ needed to reduce the turbulence to a certain value depends on the viscosity. For this reason, we studied high Stuart number cases. In these configurations, problems with the prediction of the transient flow arise and the $C F L$ condition is not enough to guarantee the convergence. Therefore an expression to estimate a value of time-step is proposed and it precisely predict and solve the aforementioned problems for high inertia problems. 


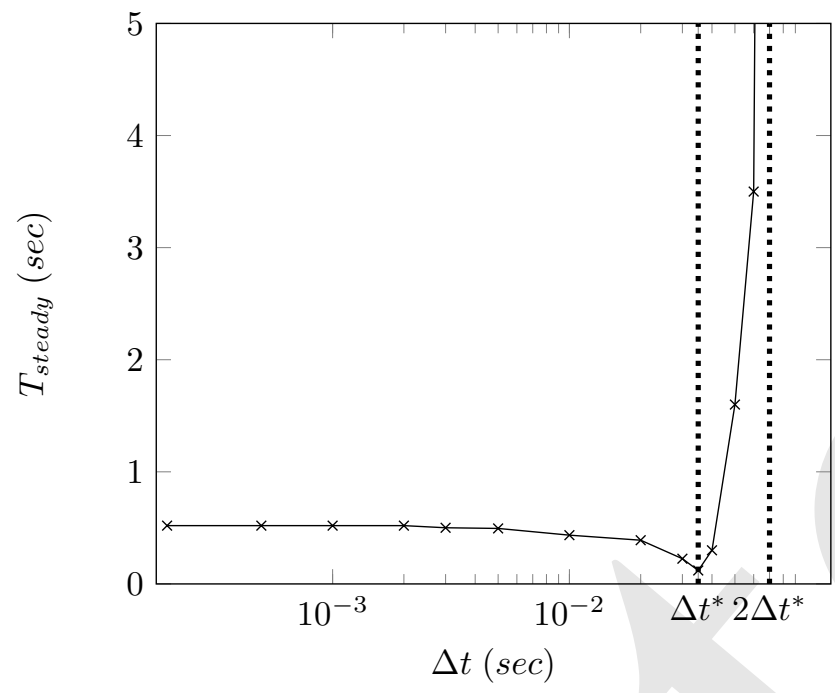

Figure 17. Physical time to reach the steady state in respect to the time-step

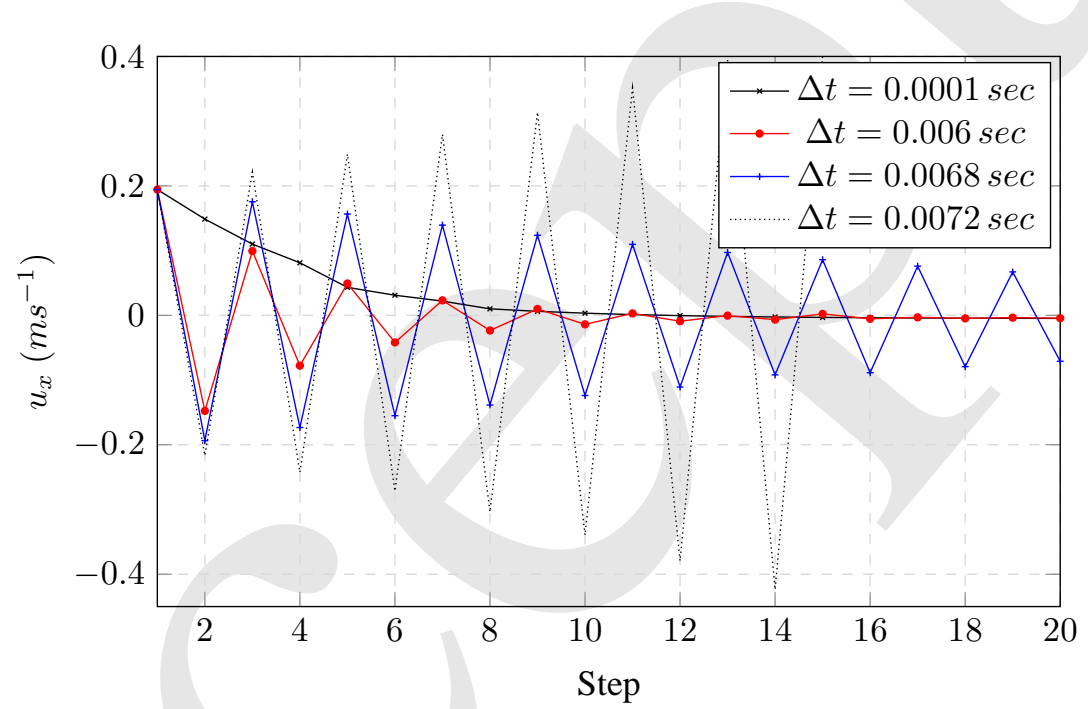

Figure 18. Evolution of the horizontal velocity at $P(0.5 ; 0.75)$ with different time-steps and $\Delta t^{*}=$ $0.0035 \mathrm{sec}$

\section{ACKNOWLEDGMENTS}

The authors give thanks to Transvalor S.A. for funding this research and for the continuous support which allowed the complete exploitation of its software. 
References

1. U. Ghia, K. N. Ghia, and C. Shin. High-resolution for incompressible flow using the navier-stokes equations and a multigrid method. Journal of computational physics, 48(3):387-411, 1982.

2. M. Sahin and N.G. Owens. A novel fully-implicit finite volume method applied to the lid-driven cavity problem. part i: high reynolds number flow calculations. International Journal for Numerical Methods in Fluids, 42:57-77, 2003.

3. J. Zhang. Numerical simulation of $2 \mathrm{~d}$ square driven cavity using fourth-order compact finite difference schemes. Computers \& Mathematics with Applications, 45(1):43-52, 2003.

4. O. Botella and R. Peyret. Benchmark spectral results on the lid-driven cavity flow. Computers \& Fluids, 27(4):421433, 1998.

5. C.H. Bruneau and M. Saad. The behaviour of high reynolds flows in a driven cavity. ColdFusion Developer's Journal (CFDJ), 15(3), 2006.

6. V. Gravemeier, W. A. Wall, and E. Ramm. A three-level finite element method for the instationary incompressible navier-stokes equations. Computer Methods in Applied Mechanics and Engineering, 193(15-16):1323-1366, 2004.

7. A. Masud and R. A. Khurram. A multiscale finite element method for the incompressible navier-stokes equations. Computer Methods in Applied Mechanics and Engineering, 195:1750-1777, 2006.

8. E. Hachem, B. Rivaux, T. Kloczko, H. Digonnet, and T. Coupez. Stabilized finite element method for incompressible flows with high reynolds number. Journal of computational physics, 229:8643-8665, 2010.

9. T. Coupez and E. Hachem. Solution of high-reynolds incompressible flow with stabilized finite element and adaptive anisotropic meshing. Computer Methods in Applied Mechanics and Engineering, 267:65-85, 2013.

10. Y. F. Peng, Y. H. Shiau, and R. R. Hwang. Transition in a 2-d lid-driven cavity flow. Computers \& Fluids, 32:337$352,2003$.

11. Y. Yu, B.W. Li, and A. Thess. The effect of a uniform magnetic field on vortex breakdown in a cylinder with rotating upper lid. Computers \& Fluids, 88:510-523, 2013.

12. E.E. Tzirtzilakis and M.A. Xenos. Biomagnetic fluid flow in a driven cavity. Meccanica, 48:187-200, 2013.

13. G.R. Kefayati. Lattice boltzmann simulation of natural convection in nanofluid-filled $2 \mathrm{~d}$ long enclosures at presence of magnetic field. Theoretical and Computational Fluid Dynamics, 27(6):865-883, November 2013.

14. V. Shatrov, G. Mutschke, and G. Gerbeth. Three-dimensional linear stability analysis of lid-driven magnetohydrodynamic cavity flow. Physics of Fluids, 15(8):2141-2151, 2003.

15. S. Sivasankaran, A. Malleswaran, Jinho Lee, and Pon Sundar. Hydro-magnetic combined convection in a lid-driven cavity with sinusoidal boundary conditions on both sidewalls. International Journal of Heat and Mass Transfer, 54:512-525, 2011.

16. A. Yu. Gelfgat and P. Z. Bar-Yoseph. The effect of an external magnetic field on oscillatory instability of convective flows in a rectangular cavity. Physics of Fluids, 13(8):2269-2278, August 2001.

17. Hakan F. Oztop, Khaled Al-Salem, and Ioan Pop. Mhd mixed convection in a lid-driven cavity with corner heater. International Journal of Heat and Mass Transfer, 54(15-16):3494-3504, July 2011.

18. J. P. Garandet, T. Alboissiere, and R. Moreau. Buoyancy driven convection in a rectangular enclosure with a transverse magnetic field. International Journal of Heat and Mass Transfer, 35(4):741-748, 1992.

19. H. Ozoe and K. Okada. The effect of the direction of the external magnetic field on the three-dimensional natural convection in a cubical enclosure. International Journal of Heat and Mass Transfer, 32:1075-1084, 1989.

20. P.X. Yu, J.X. Qiu, Q. Qin, and Z. F. Tian. Numerical investigation of natural convection in a rectangular cavity under different directions of uniform magnetic field. International Journal of Heat and Mass Transfer, 67:1131-1144, 2013.

21. L. Davoust, M.D. Cowley, R. Moreau, and R. Bolcato. Buoyancy-driven convection with a uniform magnetic field. part 2. experimental investiga- tion. Journal of Fluid Mechanics, 400(59), 1999.

22. D. Krasnov, O. Zikanov, J. Schumacher, and T. Boeck. Magnetohyfrodynamic turbulence in a channel with spanwise magnetic field. Physics of fluids, 20:095105, 2008.

23. T. Coupez. Metric construction by length distribution tensor and edge based error for anisotropic adaptive meshing. Journal of computational physics, 230:2391-2405, 2011. 